تأثير عمق النفايات الصلبة البلاية في مواقع الدفن على كمية ونوعية العصارة الناتجة

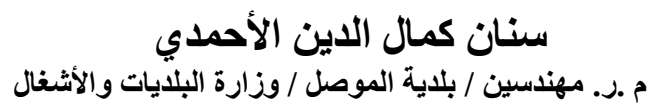

\author{
د. د. أنس فخري قاصد \\ مدرس / قسم هندسة البيئة / كلئة الهنلسة / جامعة الموصل
}

\begin{abstract}
الملخص الملإية

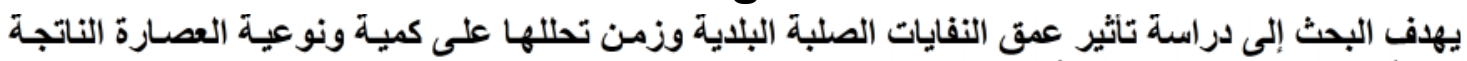

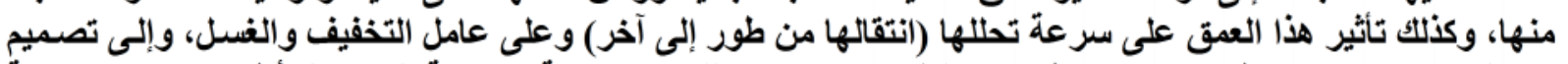

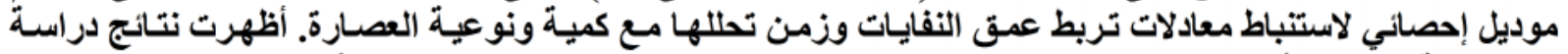

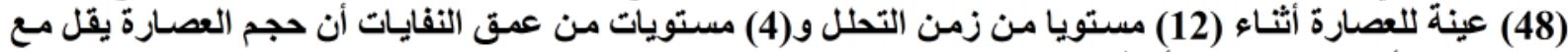

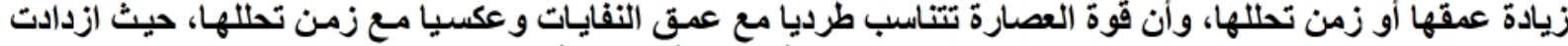

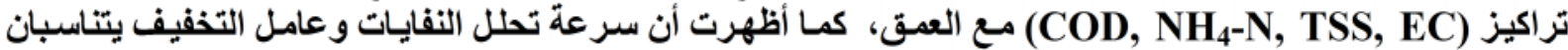

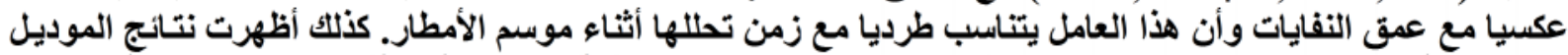

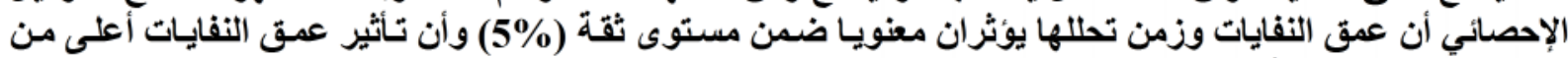

$$
\text { على نوعية العصارة. }
$$

: النفايات الصلبة البلاية ، العصارة ، عمق النفايات ، سرعة التحلل
\end{abstract}

\title{
Effect of Municipal Solid Waste (MSW) depth on leachate quantity and quality
}

\begin{abstract}
The research aimed at studying the effect of (Municipal Solid waste) depth (MSW-D) and their degradation time (MSW-T) on the quality and quantity of leachate produced (L-Q\&Q), and the effect of (MSW-D) on rapid degradation (phase converting rate) and dilution factor (D.F) (wash out during rainy seasons). It also aimed at design a statistical-model and equations to link (MSW-D) and (MSW-T) with (L-Q\&Q). A study of (48) leachate samples for $12^{\text {th }}$ (time-levels), $4^{\text {th }}$ (depth-levels) showed that the leachate volume reduced with (MSW-D) or (DT) increasing, and the leachate strength changed positively with (MSW-D) and inversely with (DT) so the (COD, $\left.\mathrm{NH}_{4}-\mathrm{N}, \mathrm{TSS}, \mathrm{EC}\right)$ concentrations were increased with (MSW-D). It also showed that the rapidity degradation and (D.F) changed inversely with (MSW-D), and that (D.F) increased with increasing of (MSW-T) during rainy seasons. The statistical-model results showed that the (MSWD) and (MSW-T) were significantly affected (within-5\% confidence-level) on (L-Q\&Q), and that (MSW-D) was more effective than (MSW-T).
\end{abstract}




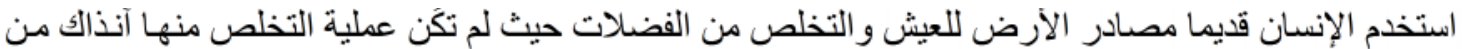

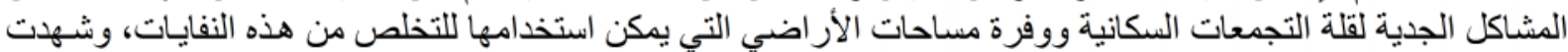

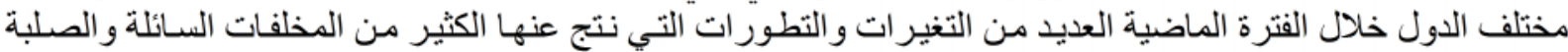

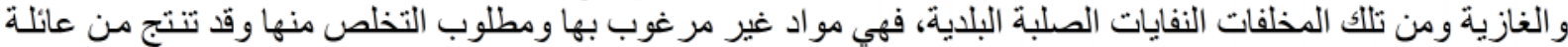

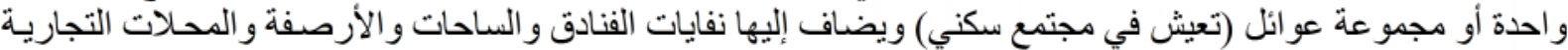

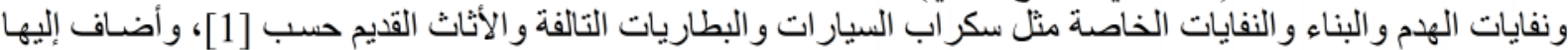

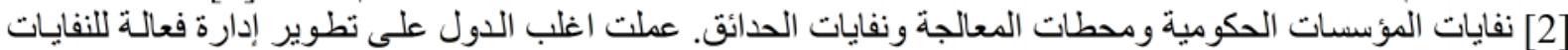

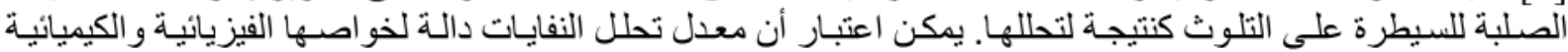

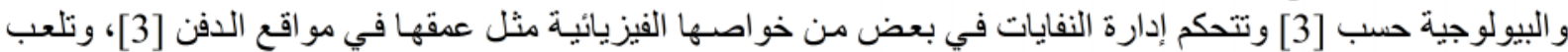

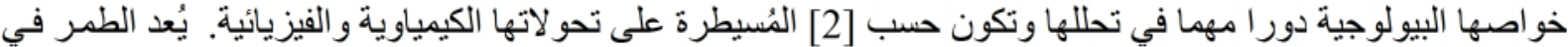

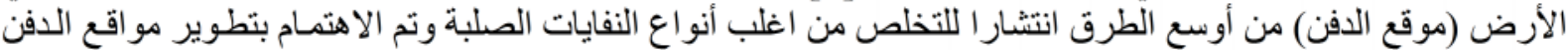

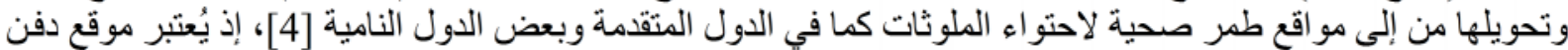

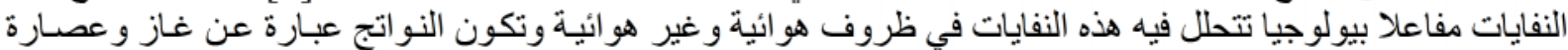

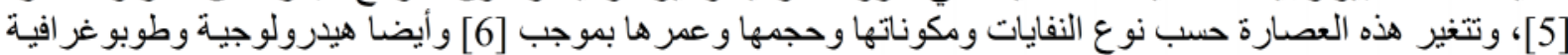

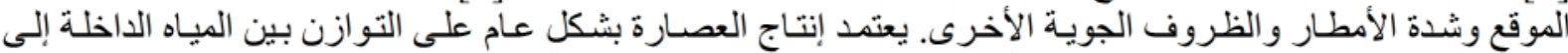

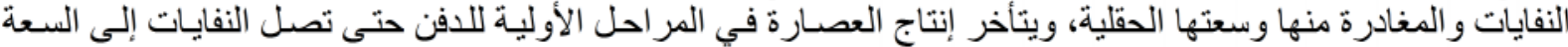

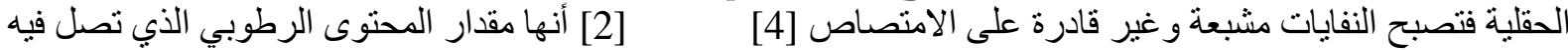

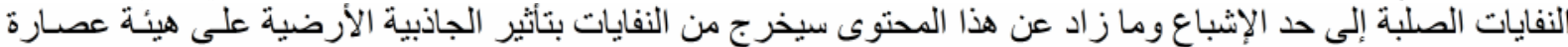

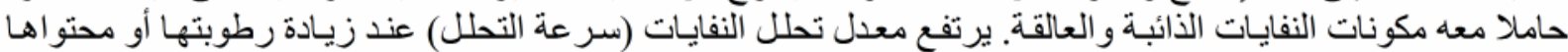

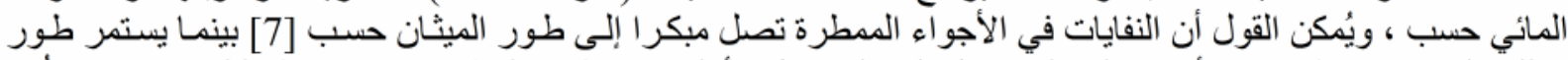

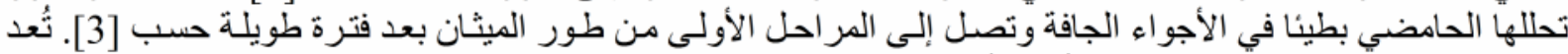

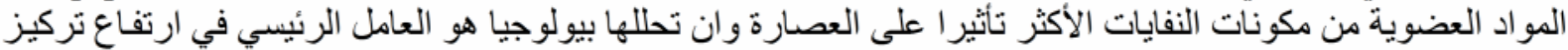

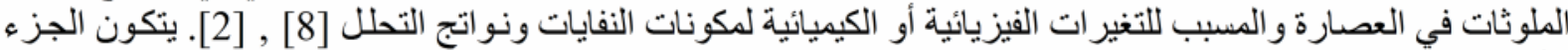

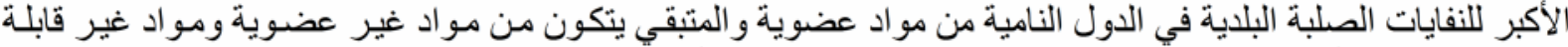

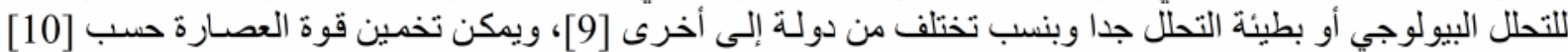

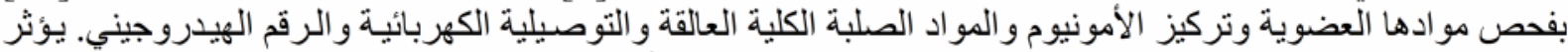

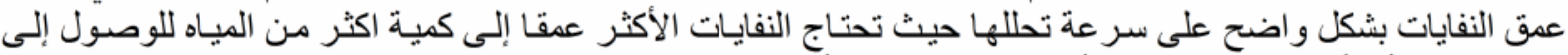

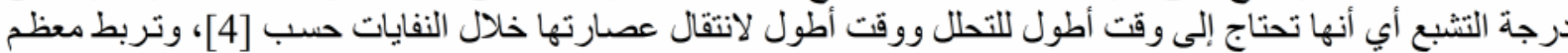

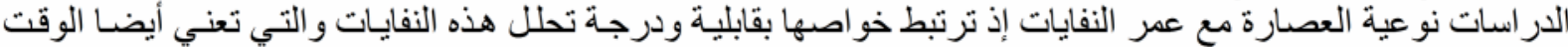

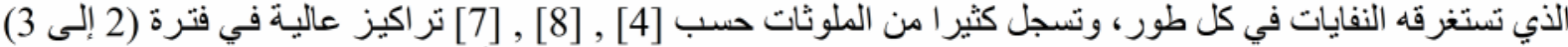

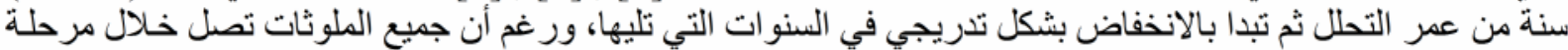

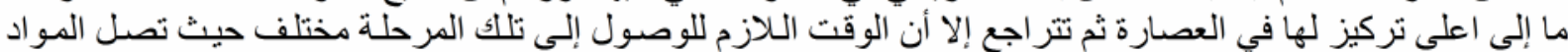

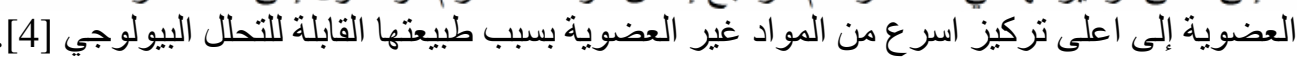

أهداف البحث

در اسة تأثنير عمق النفايات الصلبة البلدية على كمية ونوعية العصارة الناتجة.

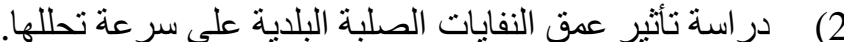

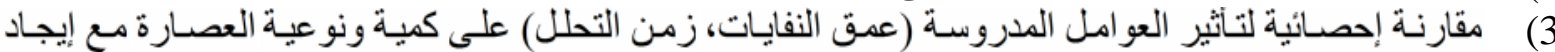
علاقات إحصائية رياضية.

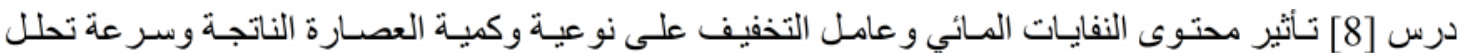

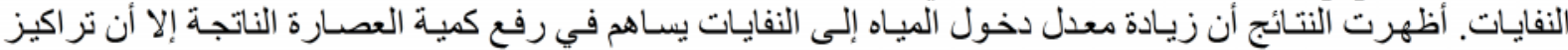

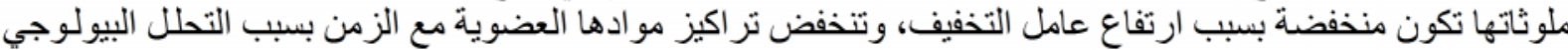
السريع للنفايات الحديثة الدفن. 
ودرس [7 [أثثير عامل التخفيف وزمن تحلل النفايات على خو اص العصارة الناتجـة ومقارنتها ببر امج إحصـائية.

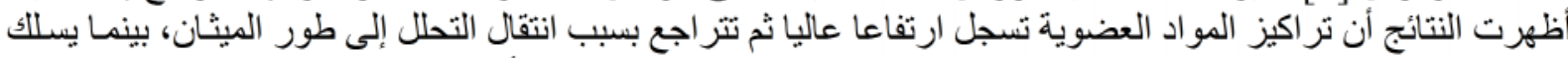

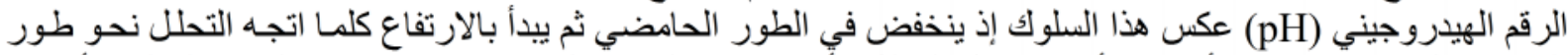

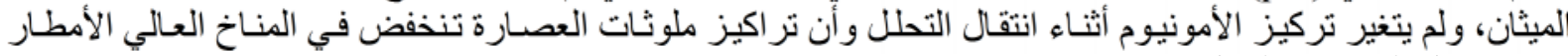
بسبب عامل التخفيف و الغسل.

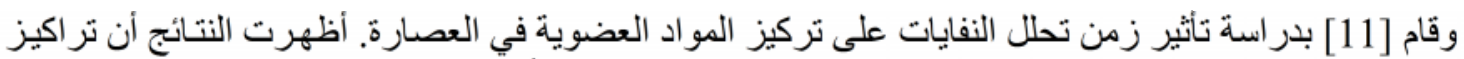

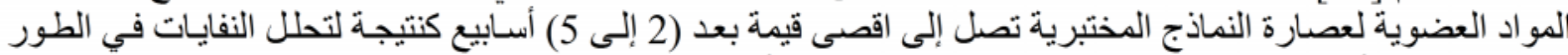

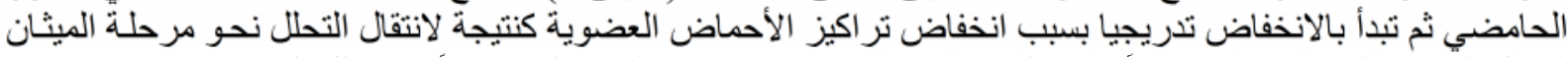
لتصل إلى مرحلة الاستقرار في الأسبوع السابع عشر كنتيجة لاستنز اف المواد الداد العضوية أثناء تحلل النفايات.

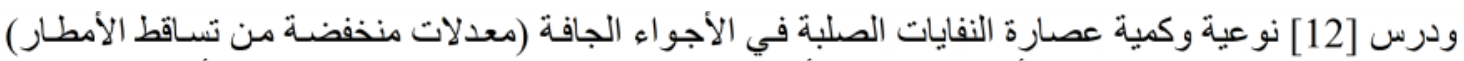

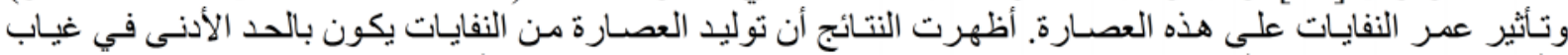

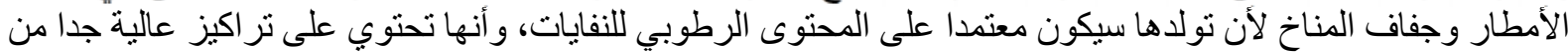

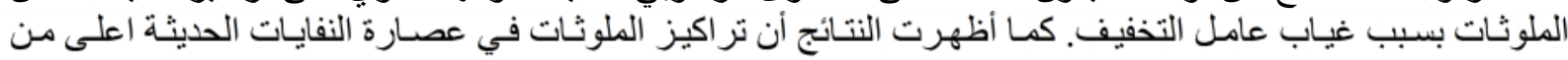

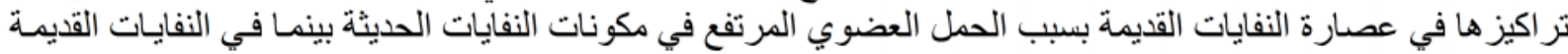

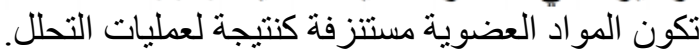

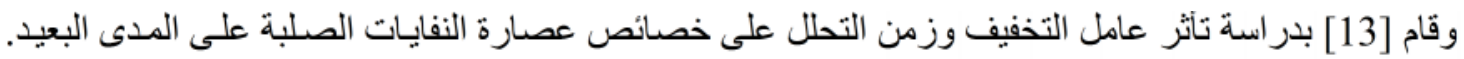

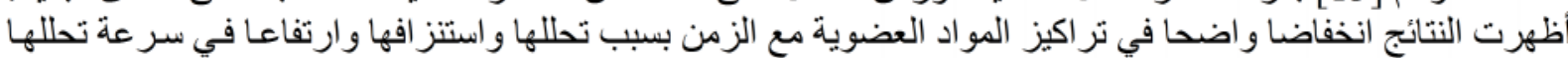

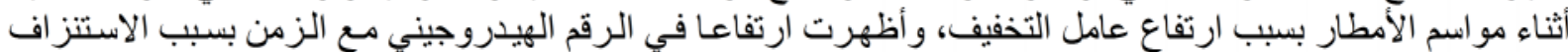

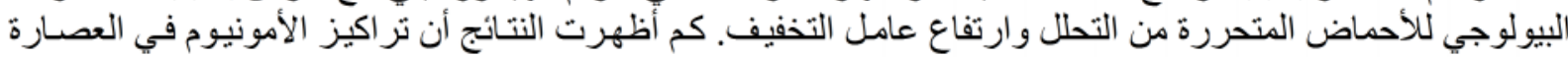

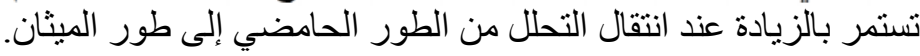

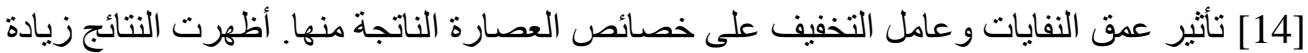

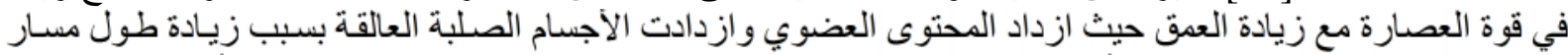

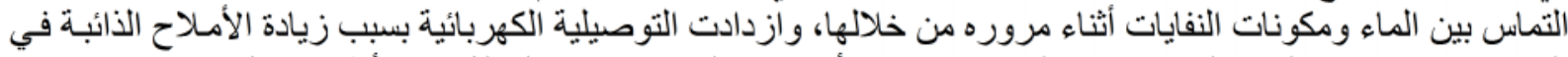

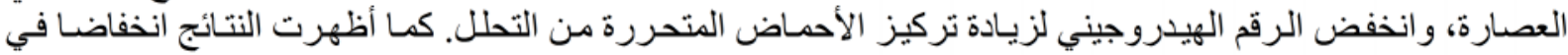

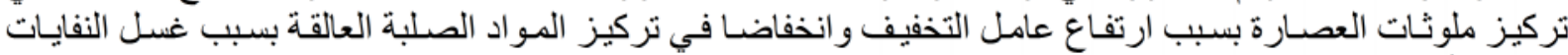
المستمر أثناء مرور الماء من خلالها.

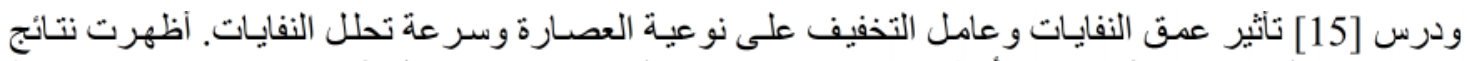

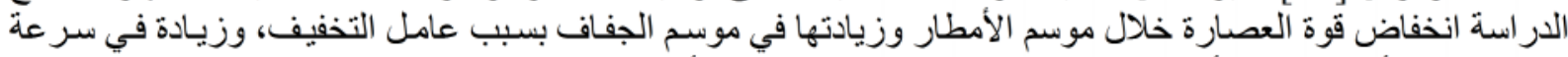

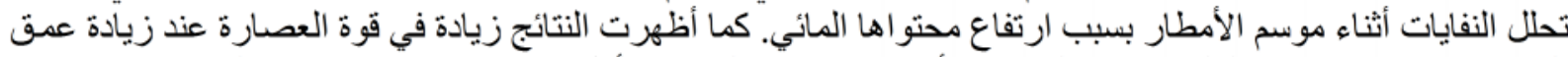

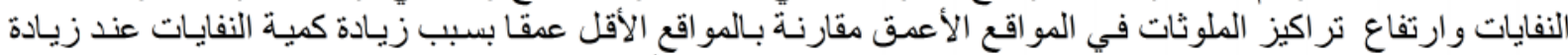

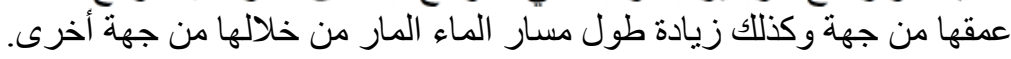

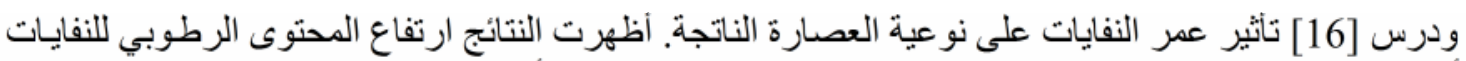

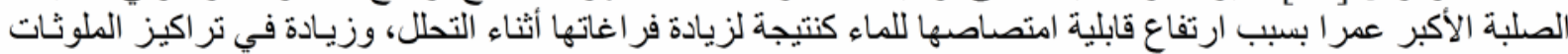

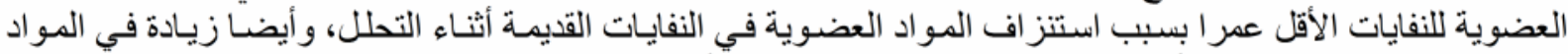

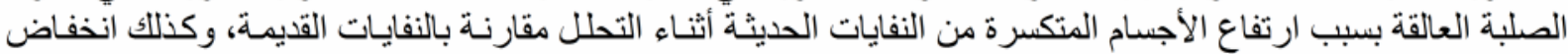

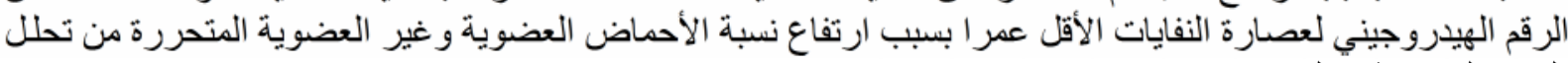

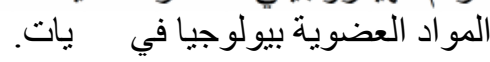

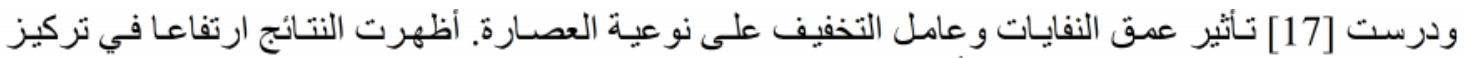

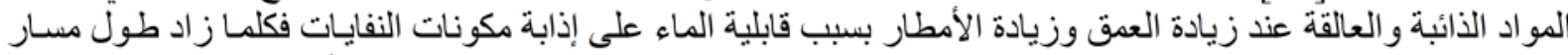

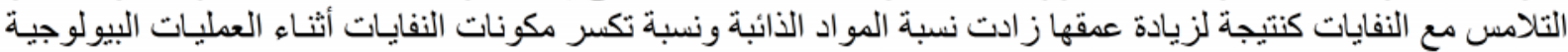

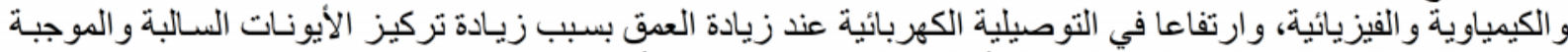

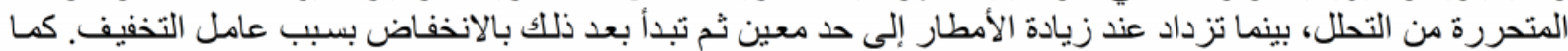

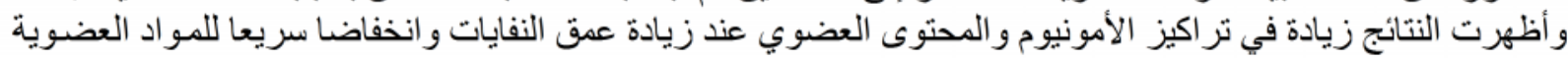


بالأمو نيوم عند زيادة الأمطار بسبب زيادة سر عة تحلل المكونات العضوية كنتيجة لارتفاع محتو اها المسائي من جهة

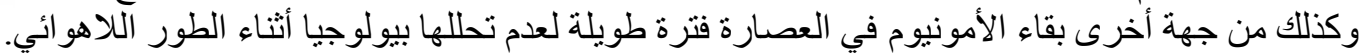

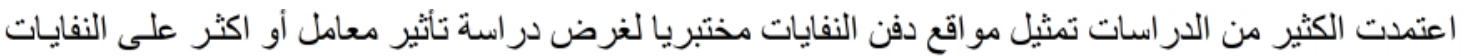

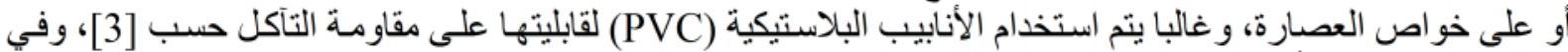

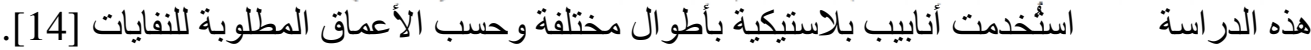

(1) - تهيئة النفايات الصلبة البلاية

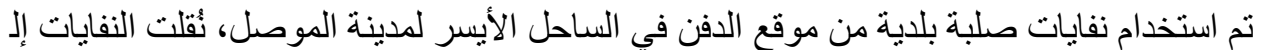

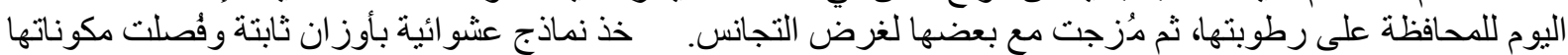

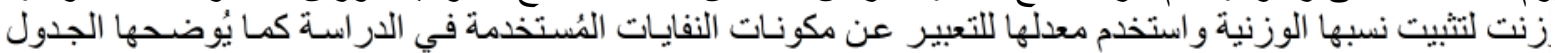

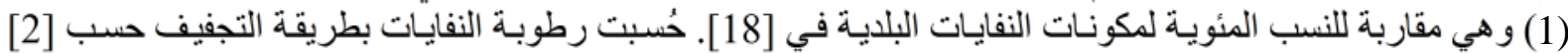

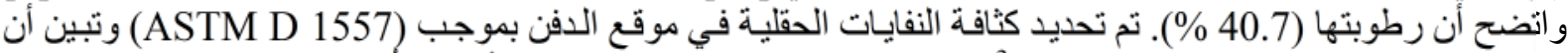

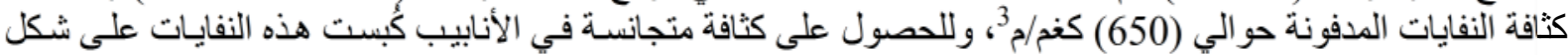

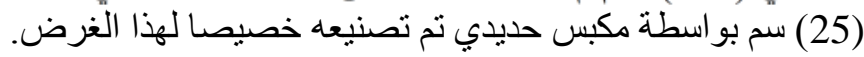

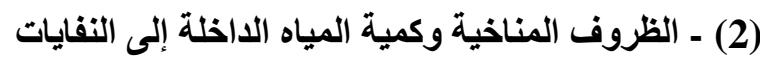

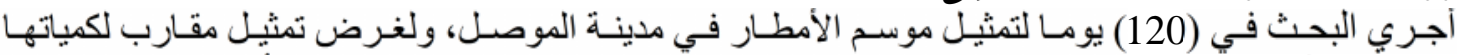

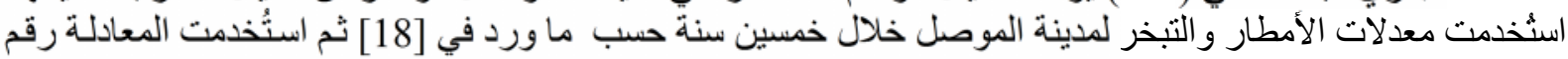

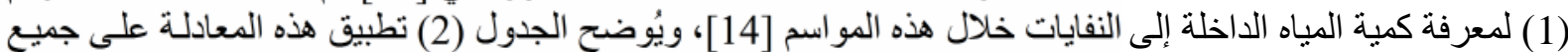
الأشهُر حيث يمثل الرقم الموجب كمية المياه النافذة ويمثل الرقم السالب كمية المياه المتبخرة.

(1) مكونات النفايات الصلبة البلدية

\begin{tabular}{|c|c|c|c|c|c|c|}
\hline \multirow{2}{*}{ No } & Components & Weight \% & \multirow{2}{*}{ No } & \multicolumn{2}{|c|}{ Components } & Weight \% \\
\cline { 2 - 3 } \cline { 5 - 6 } & \multicolumn{2}{|c|}{ Organic } & & \multicolumn{2}{|c|}{ Inorganic } \\
\hline 1 & Food Wastes & 66.8 & & 10 & Glass & 2.40 \\
\hline 2 & Cardboards & 5.9 & & 11 & Tin Cans & 2.30 \\
\hline 3 & Paper & 4.15 & & 12 & Aluminum & 0.40 \\
\hline 4 & Plastics & 5.70 & & 13 & Other metal & 0.90 \\
\hline 5 & Textiles & 6.10 & & 14 & Hazardous Waste & 0.70 \\
\hline 6 & Rubbers & 1.10 & & 15 & Dirt, Ashes, etc. & 0.90 \\
\hline 7 & Leather & 0.20 & \multicolumn{3}{|}{} \\
\hline 8 & Trimmings & 2.10 & \multicolumn{3}{|c}{} \\
\hline 9 & Woods & 0.40 & \multicolumn{3}{|c}{} \\
\end{tabular}

(2) محصلة المياه النافة خلال فصول السنة

\begin{tabular}{|c|c|c|c|c|c|c|c|}
\hline Months & $\begin{array}{c}\text { Rain } \\
(\mathrm{mm})\end{array}$ & $\begin{array}{c}\text { Evaporation } \\
(\mathrm{mm})\end{array}$ & $\begin{array}{c}\mathrm{H}=\mathrm{P}(1-\mathrm{C})-\mathrm{E} \\
(\mathrm{mm})\end{array}$ & Months & $\begin{array}{c}\text { Evaporation } \\
(\mathrm{mm})\end{array}$ & $\begin{array}{c}\text { Rain } \\
(\mathrm{mm})\end{array}$ & $\begin{array}{c}\mathrm{H}=\mathrm{P}(1-\mathrm{C})-\mathrm{E} \\
(\mathrm{mm})\end{array}$ \\
\hline January & 62 & 5.8 & 46.9 & July & - & 233.9 & -233.9 \\
\hline February & 70 & 10.8 & 48.7 & August & - & 223.0 & -223.0 \\
\hline March & 75.9 & 24.1 & 40.4 & September & 0.3 & 153.0 & -152.7 \\
\hline April & 54.6 & 50.1 & -3.7 & October & 11 & 69.9 & -60.6 \\
\hline May & 23 & 111.6 & -92.1 & November & 43.7 & 25.5 & 11.6 \\
\hline June & 0.23 & 187.5 & -187.3 & December & 68.4 & 10.5 & 47.6 \\
\hline
\end{tabular}


$H=P(1-C)-E$

\section{Where:}

(H) depth of percolation water, (P) rain water, (C) runoff coefficient, (E) evaporation
$C=0.15[20][21]$
$([18]$
Silty clay
( بيث أن تربة الموقع (E) ( )

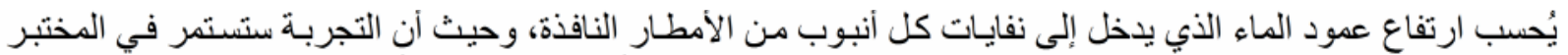

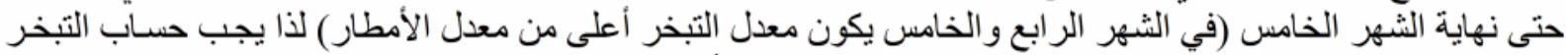

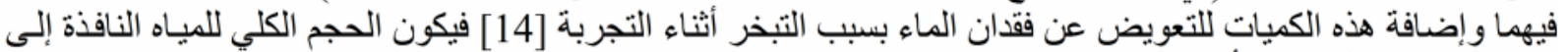

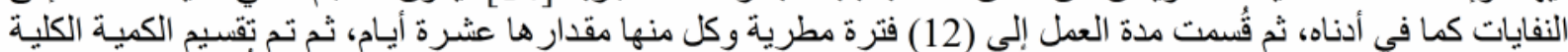

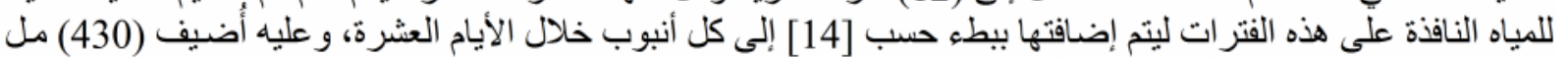
(10)

$H($ Percolation $)=(46.9+48.7+40.415+11.645+47.64)=>195.3 \mathrm{~mm}$

$H($ April $)=-3.69 \mathrm{~mm} \quad \& \quad H($ May $)=-92.05 \mathrm{~mm}$

Then $H($ Total $)=195.3+3.69+92.05=>291.04 \mathrm{~mm}$

$Q($ Total $)=291.04 \times 0.0177=>5.15$ Litter $\quad\left(\right.$ where section area $\left.=0.0177 \mathrm{~m}^{2}\right)$

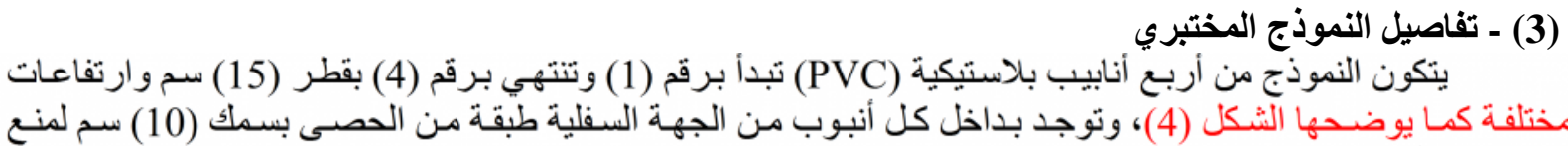

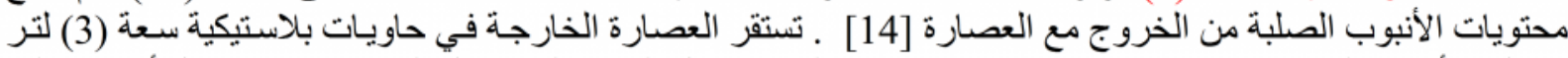

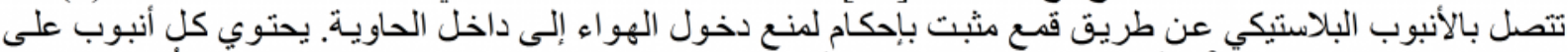

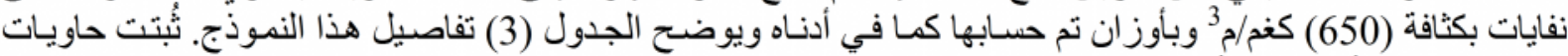

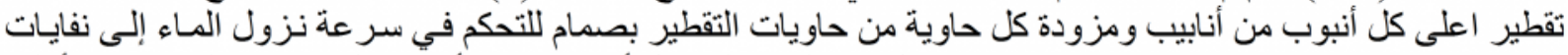

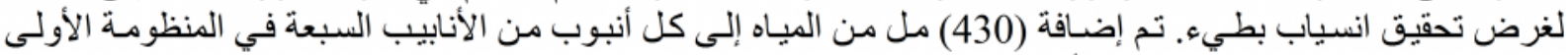

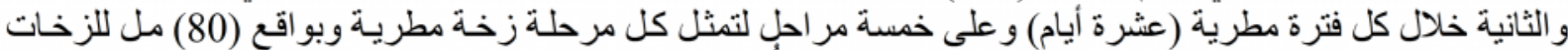

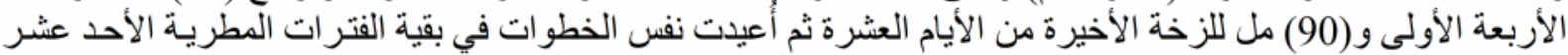

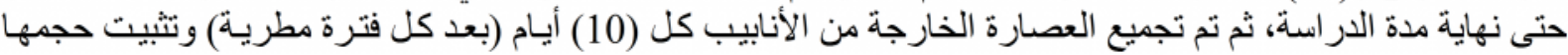

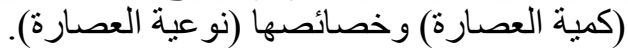

Weight of MSW = Section area of pipe $x$ Height of MSW $x$ Density of MSW

(3) (3) أنابيب المنظومة الأولى (1)

\begin{tabular}{|c|c|c|c|c|c|c|}
\hline $\begin{array}{c}\text { Column } \\
\text { No. }\end{array}$ & $\begin{array}{c}\text { Diameter } \\
(\mathrm{m})\end{array}$ & $\begin{array}{c}\text { Surface Area } \\
\left(\mathrm{m}^{2}\right)\end{array}$ & $\begin{array}{c}\text { Depth } \\
(\mathrm{m})\end{array}$ & $\begin{array}{c}\text { Volume } \\
\left(\mathrm{m}^{3}\right)\end{array}$ & $\begin{array}{c}\text { Density } \\
\left(\mathrm{Kg} / \mathrm{m}^{3}\right)\end{array}$ & $\begin{array}{c}\text { Weight } \\
(\mathrm{Kg})\end{array}$ \\
\hline 1 & 0.15 & 0.0177 & 0.5 & 0.0088 & 650 & 5.74 \\
\hline 2 & 0.15 & 0.0177 & 1.0 & 0.0177 & 650 & 11.49 \\
\hline 3 & 0.15 & 0.0177 & 1.5 & 0.0265 & 650 & 17.23 \\
\hline 4 & 0.15 & 0.0177 & 2.0 & 0.0353 & 650 & 22.97 \\
\hline
\end{tabular}

(1) - تأثير عمق النفايات على كمية العصارة

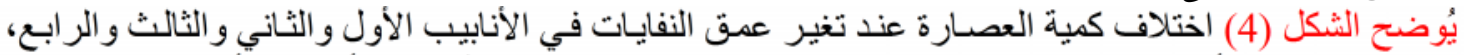

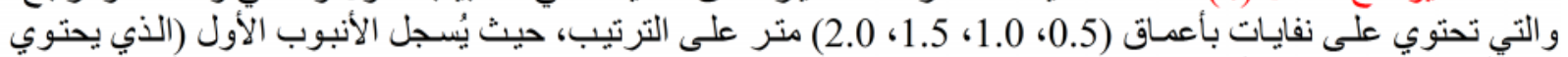

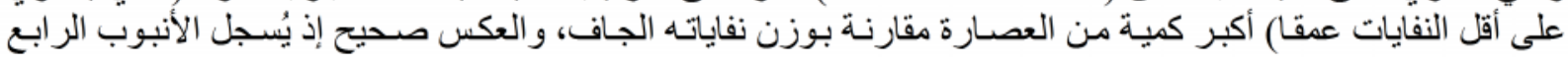




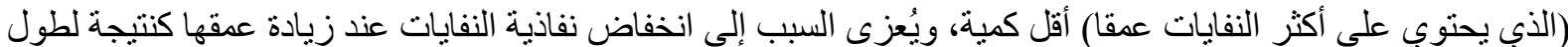

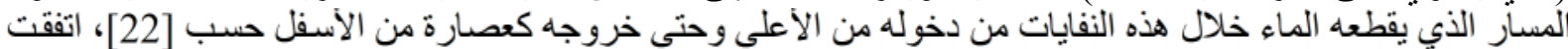

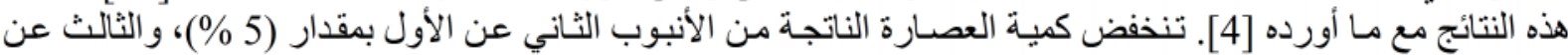

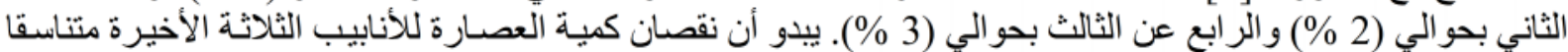

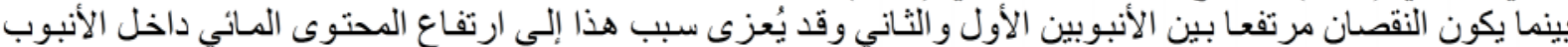

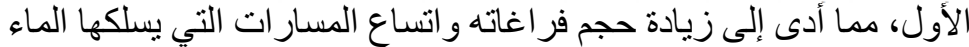

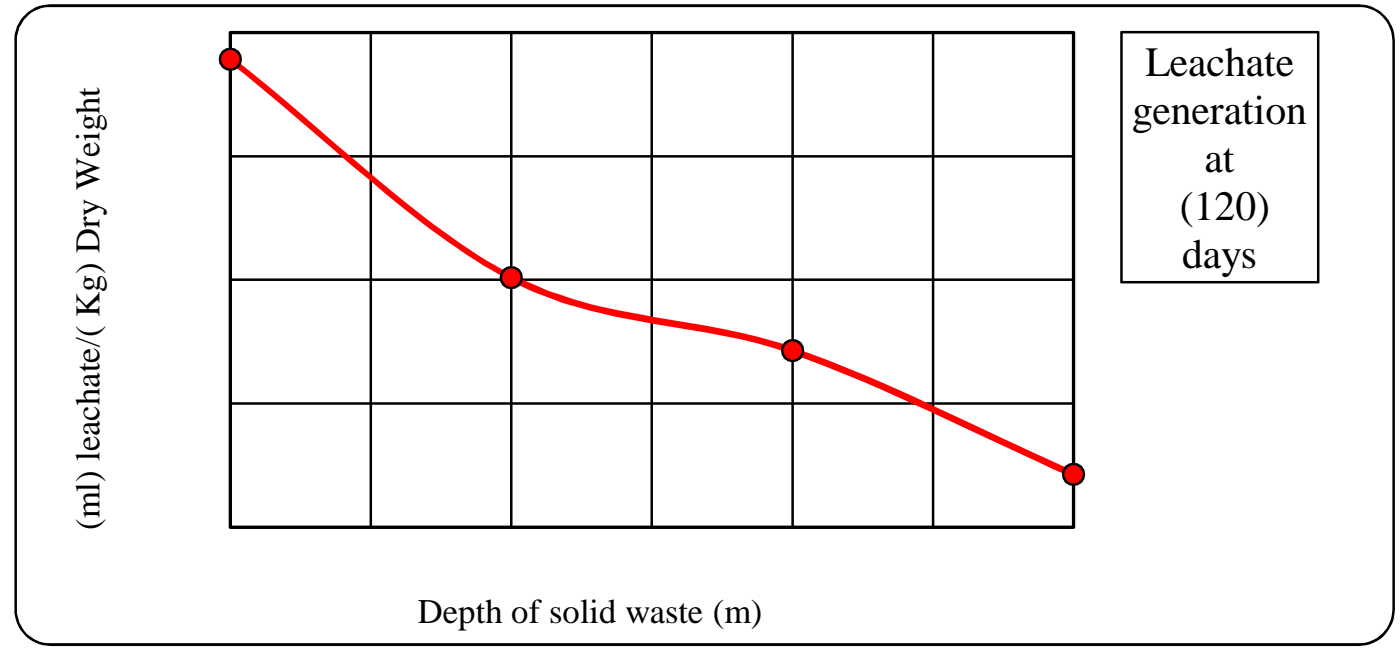

(4) ت تغاير كمية العصارة مع تغير عمق النفايات

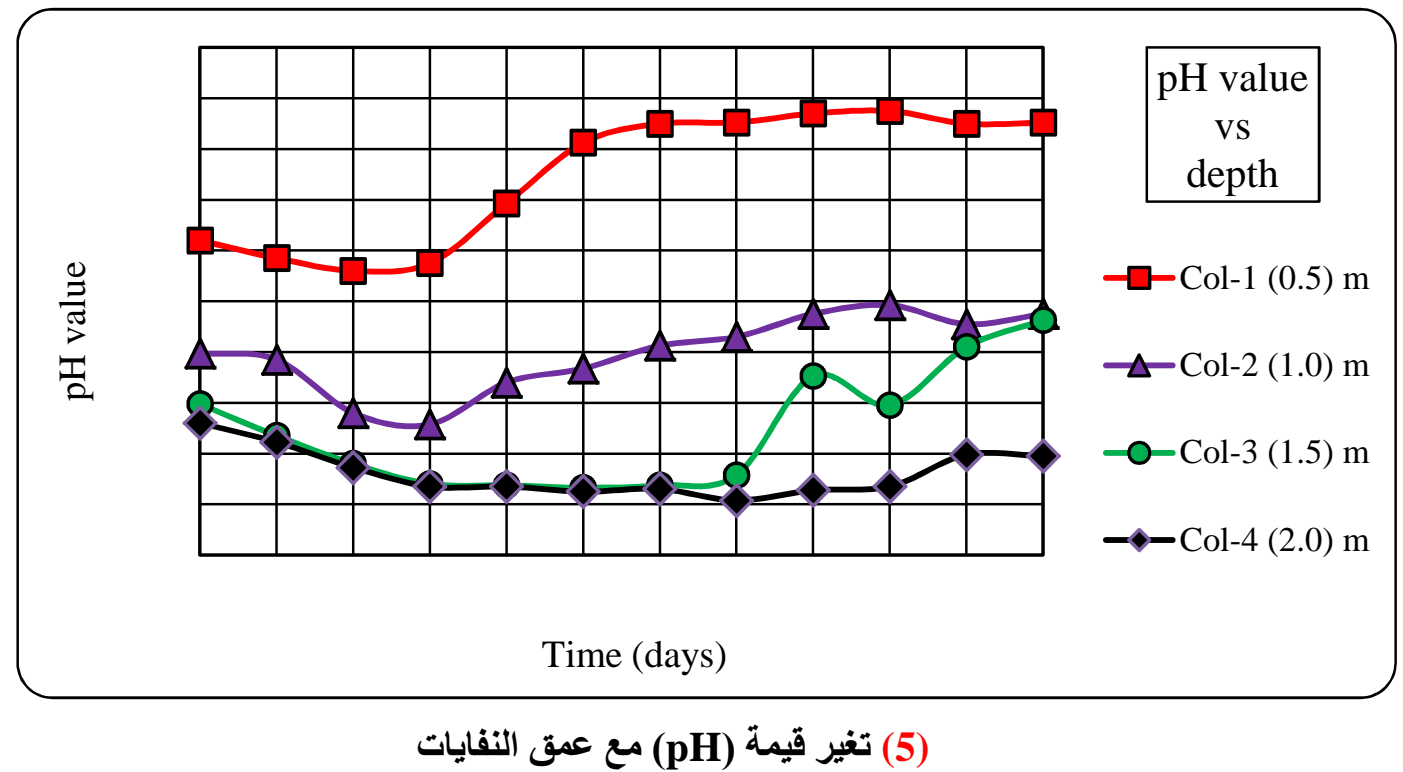

(2) ـ تأثير عمث النفايات على خواص العصارة

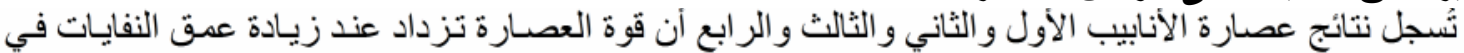

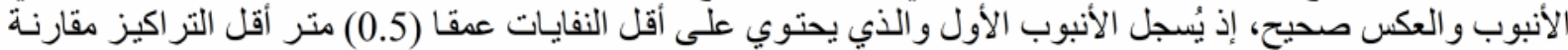

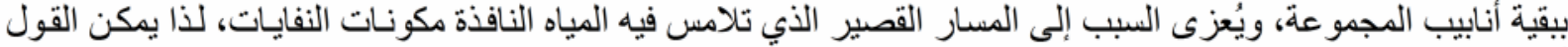

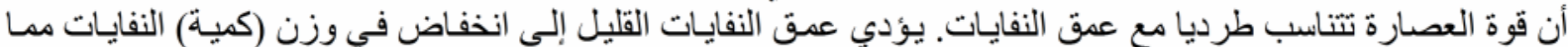

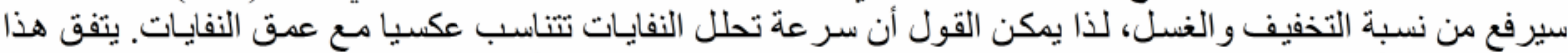
التحليل مع ما أورده في هذا المجال كل من [17] , [14] , [4] وفيما يلي نتائج تأثير العمق على نوعية 


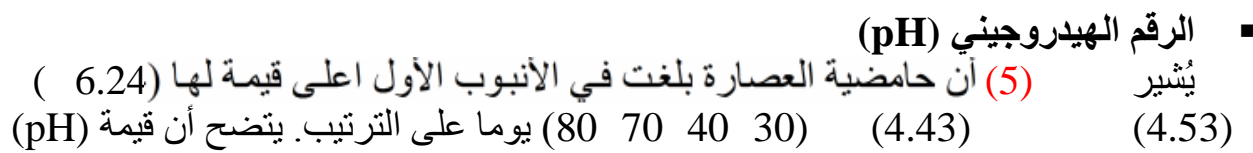

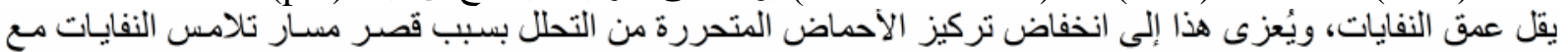

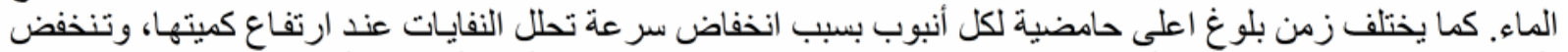

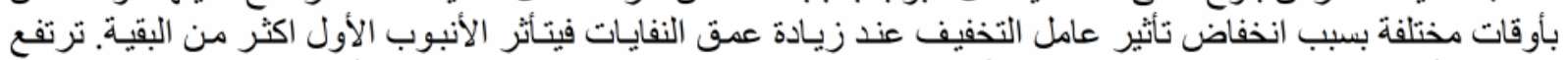

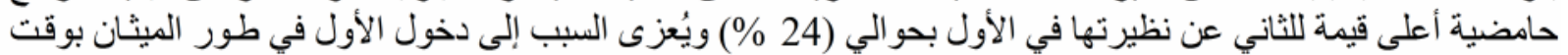

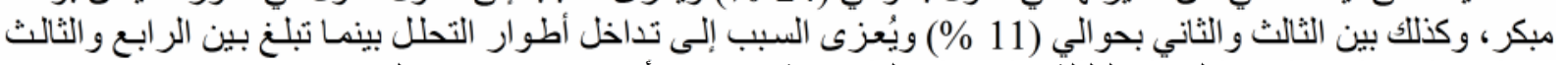

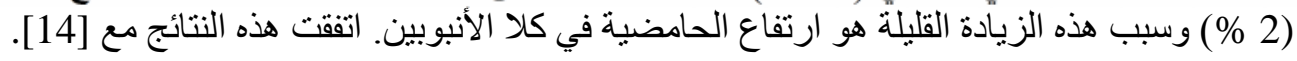

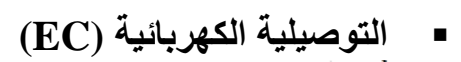

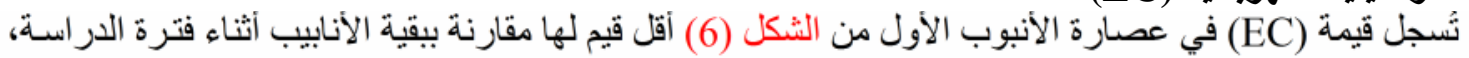

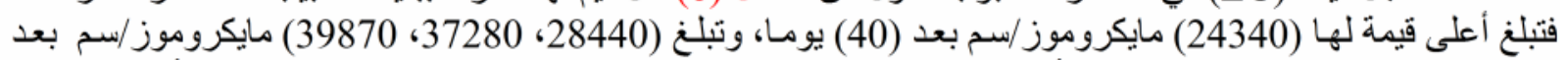

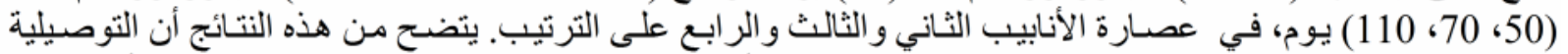

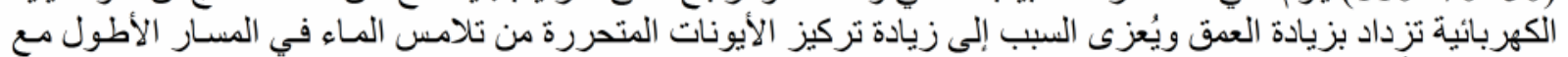

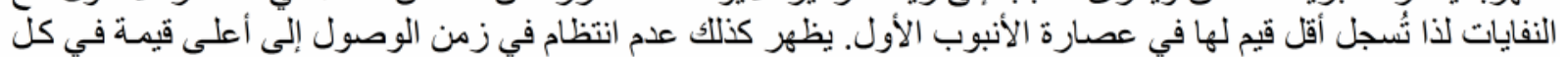

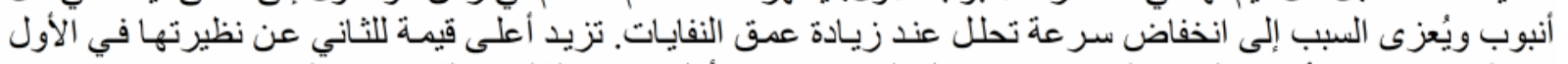

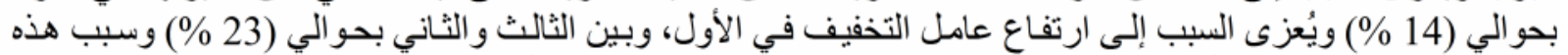

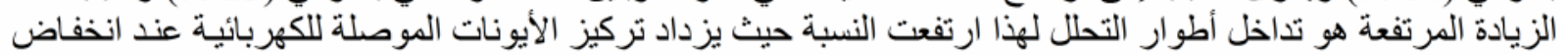

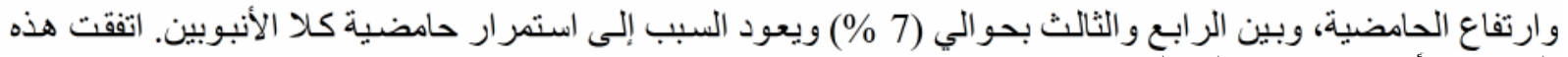

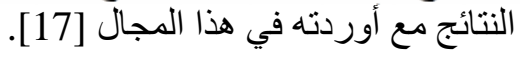

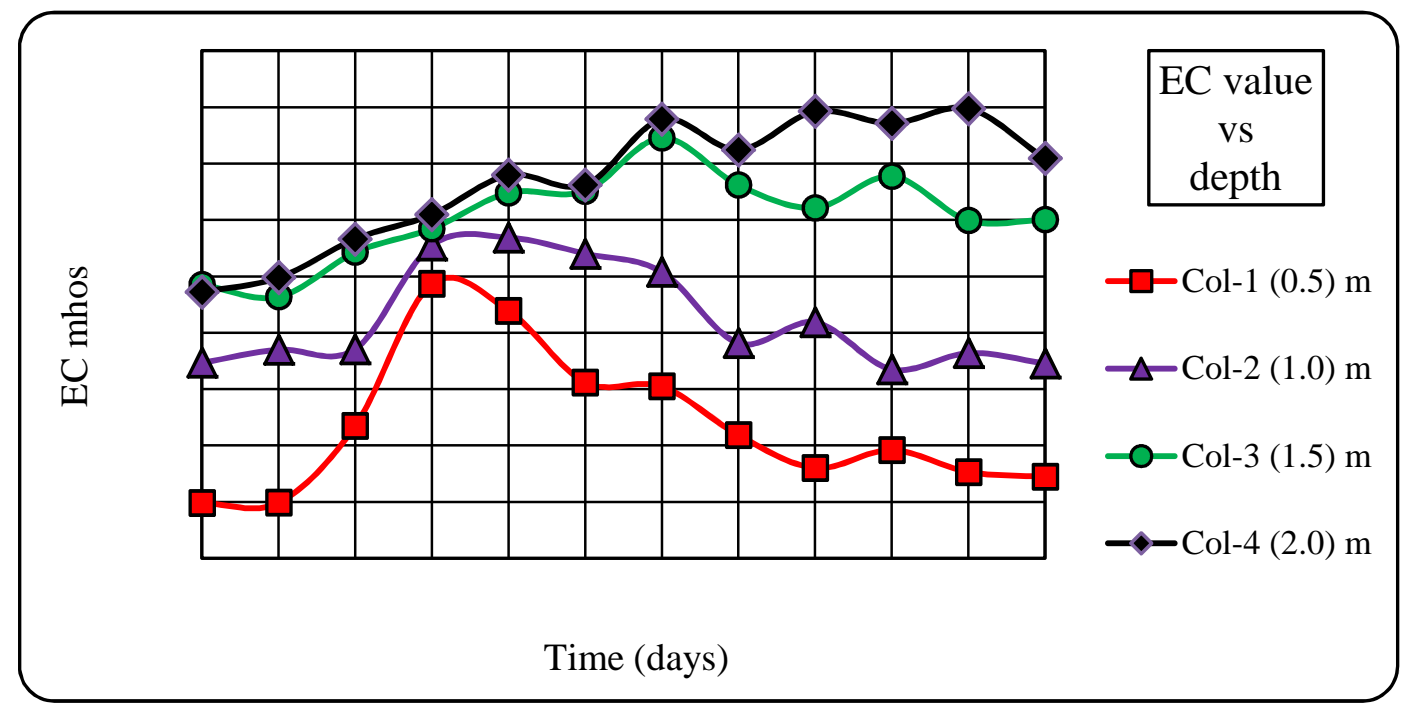

(6) تغير التوصيلية الكهربائية مع عمث النفايات

(TSS)

يُشير (7) أن تر اكيز الأجسام الصلبة الكلية العالقة بلغت أعلى قيمة ومقدار ها (687.8 1109.9

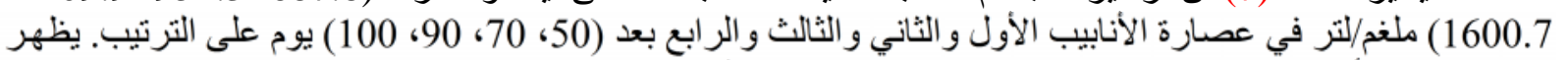

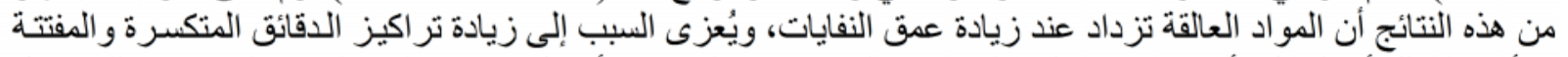

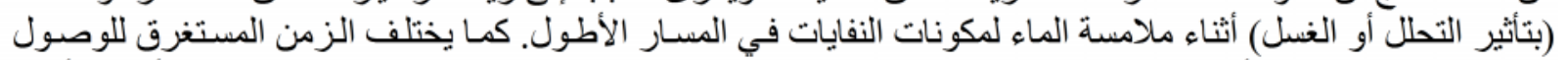

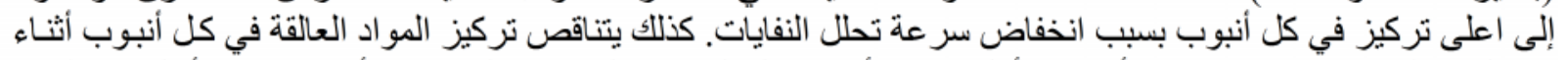

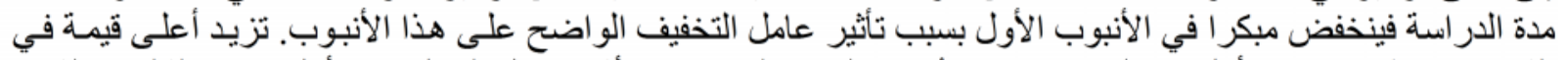

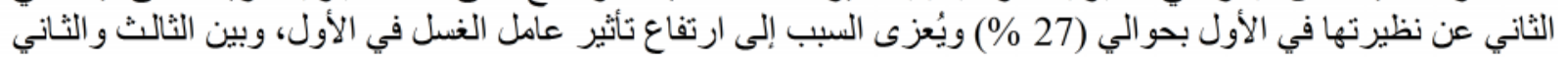




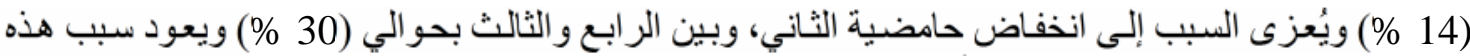

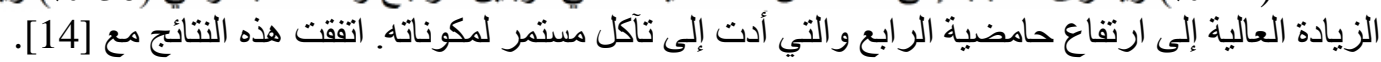

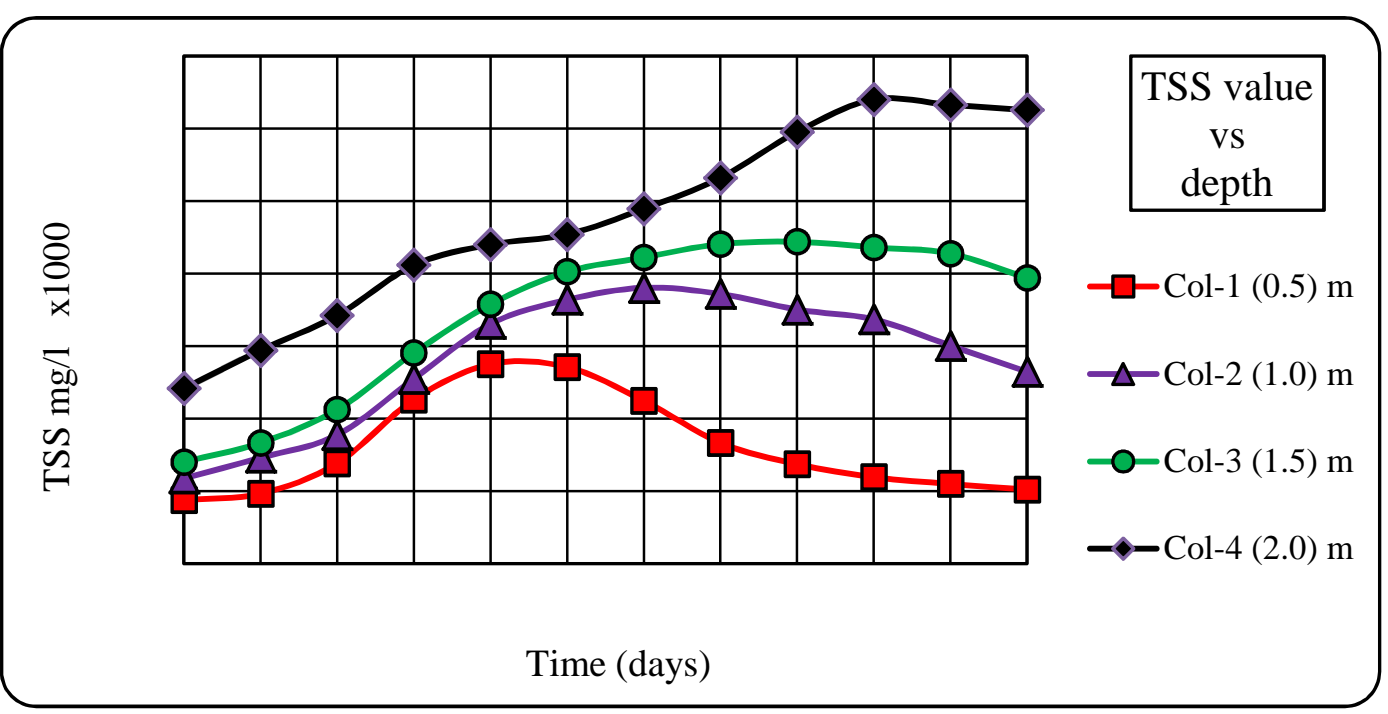

(7) تغير تركيز الأجسام الصلبة الذائبة مع كثافة النفايات

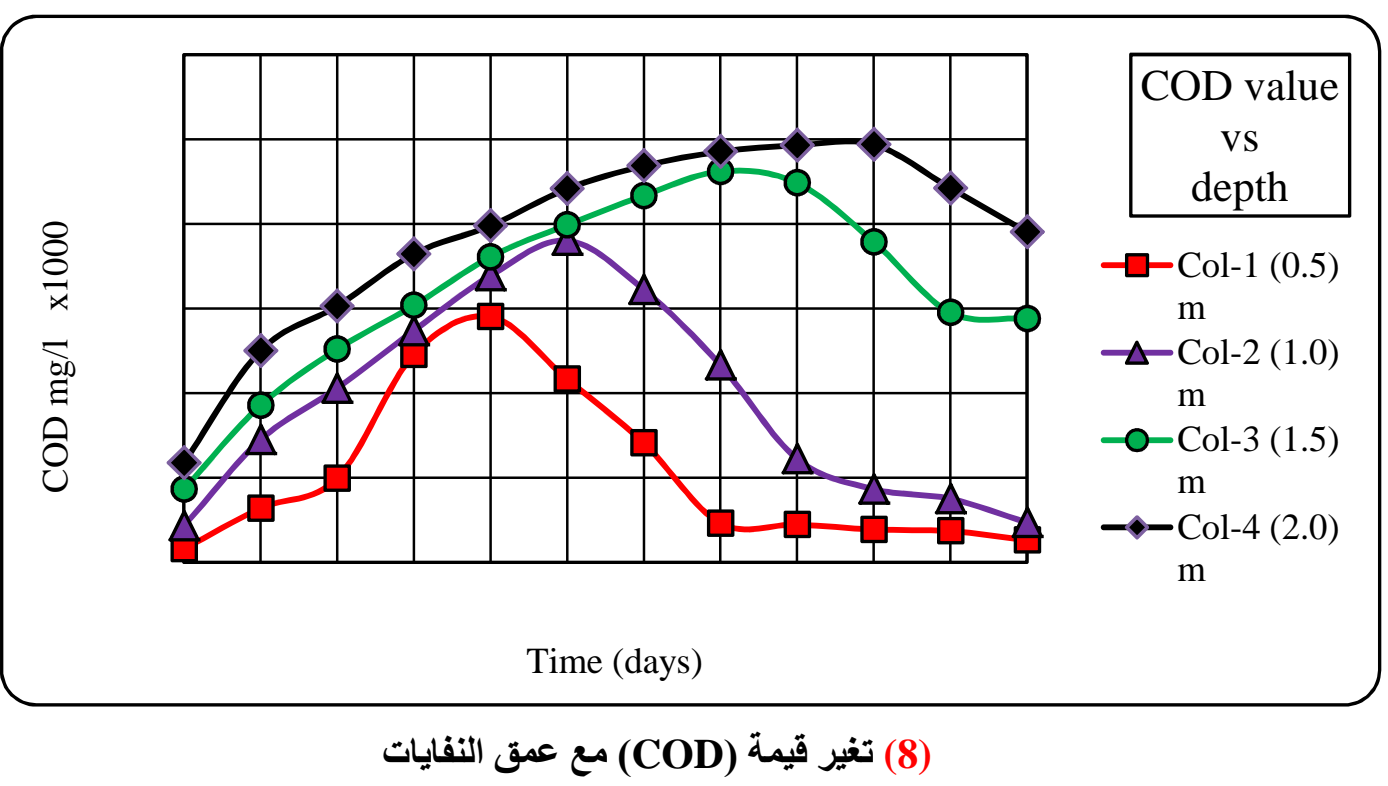

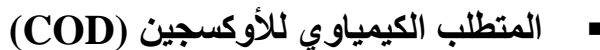

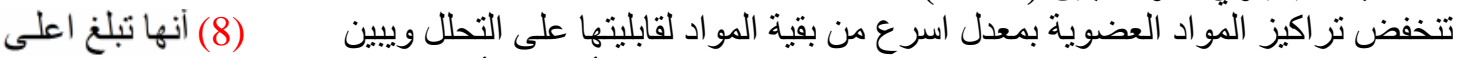

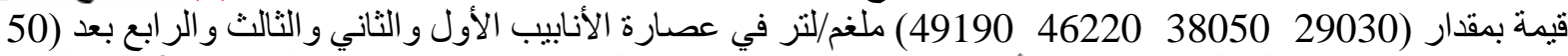

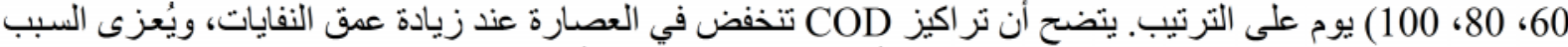

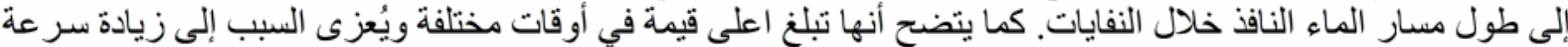

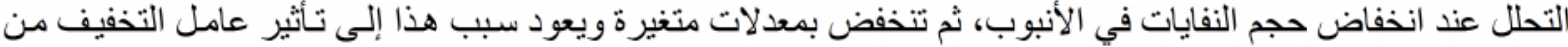

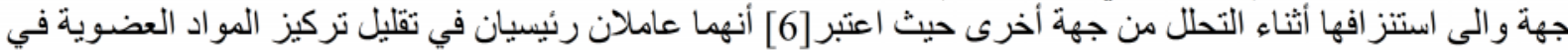

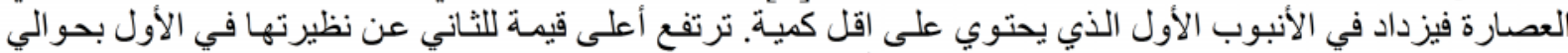

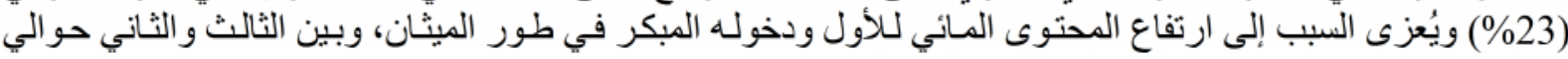


(17\%) ويُعزى السبب إلى تداخل أطو ار التحلل، وبين الر ابع والثالث حو الي (6 \%) سبب الزيادة القليلة يعود إلى ارتفاع

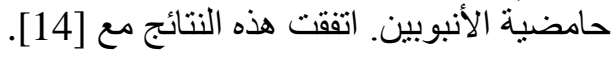

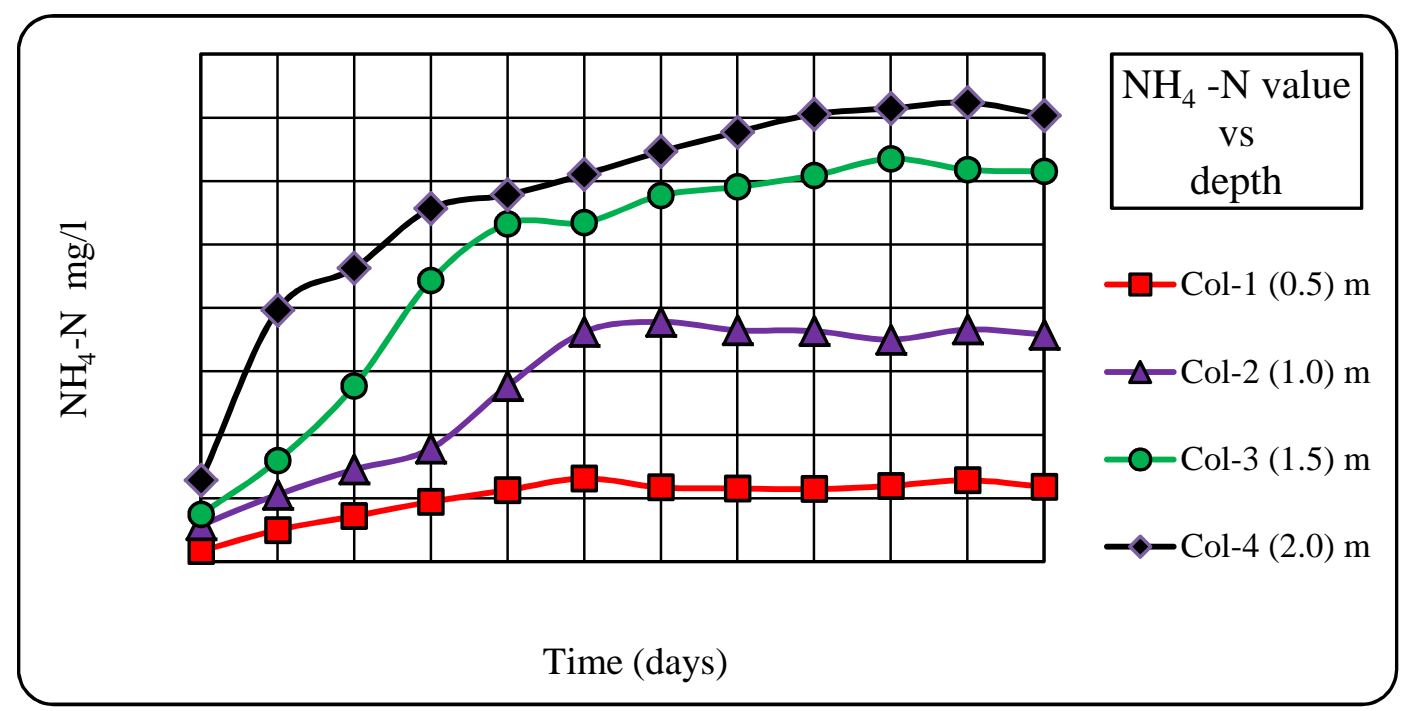

(9) تغير الأمونيوم مع عمق النفايات

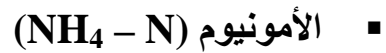

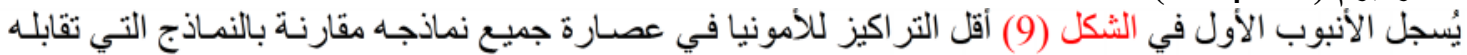

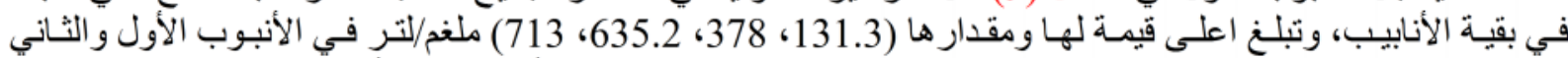

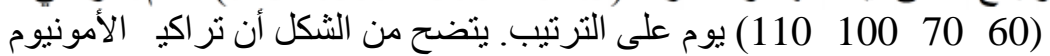

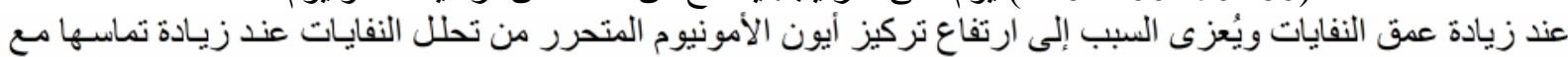

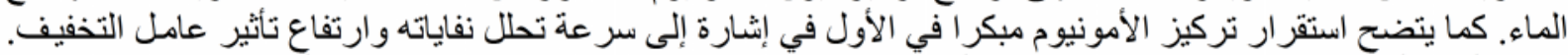

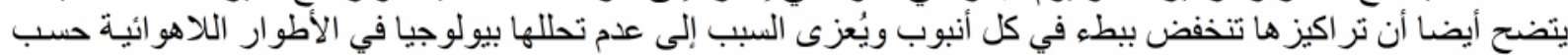

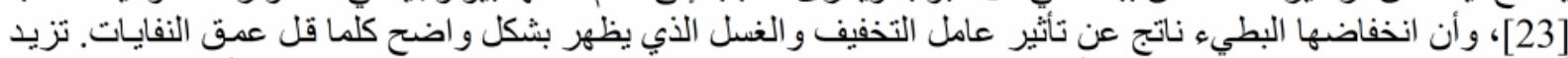

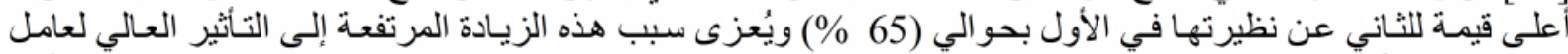

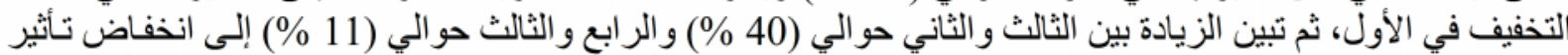

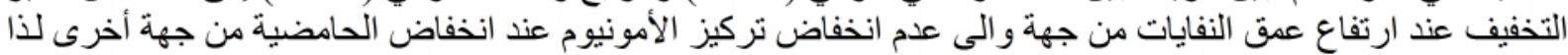

تعتبر من الملوثات الطويلة الأمد. تتفق هذه النتائج مع ما وعة ورد في [15] , [17].

(3) - الموديل الإحصائي لتأثير المتغيرات على كمية وقوة العصارة

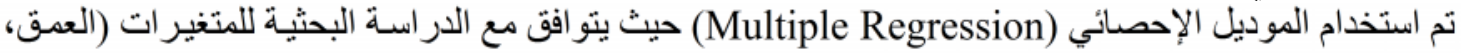

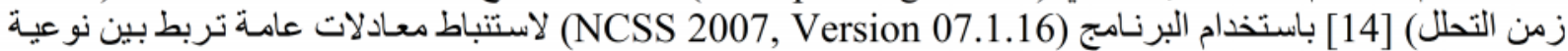

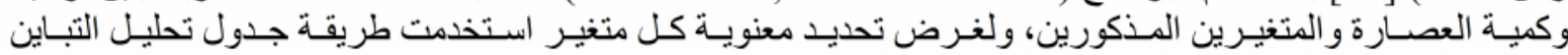

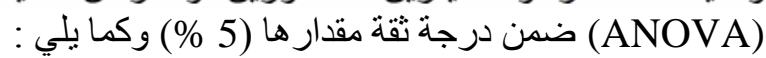

\section{-}

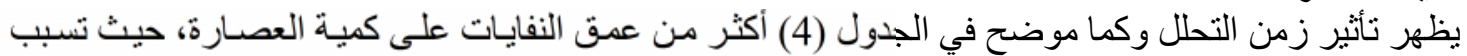

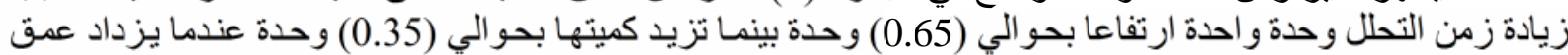

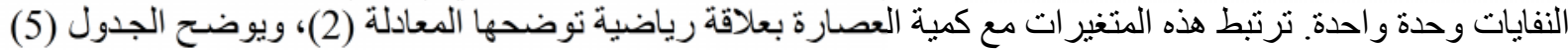
أن كل من زمن التحلل و عمث النفايات يؤثر ان معنويات على على كمية العصارة الناتجة. 
(4) تأثير العمق وزمن التحلل على كمية

\begin{tabular}{|c|c|c|}
\hline \multirow{4}{*}{ Ln (Leachate) } & Independent variable & Standardized Coefficient $(\beta)$ \\
\cline { 2 - 3 } & Intercept & 0 \\
\cline { 2 - 3 } & Ln(depth) & 0.351 \\
\cline { 2 - 3 } & $1 /$ time & 0.651 \\
\hline
\end{tabular}

Ln $($ Leachate $)=5.58+0.16 \times \operatorname{Ln}($ depth $)+0.214 \times \operatorname{Ln}($ time $) \ldots$ (2)

(5) تحليل التباين للمتغيرات ومعنوية تأثيرها على كمية العصارة

\begin{tabular}{|c|c|c|c|c|c|}
\hline Model Term & $\mathrm{DF}$ & $\mathrm{R}^{2}$ & F Calculate & Prob Level & \\
\hline Model Ln (leachate) & 2 & 0.5473 & 27.207 & $\%$ Less than 1 & \\
\hline Ln (depth) & 1 & 0.1232 & 12.247 & $\%$ Less than 1 & Significant \\
\hline Ln (time) & 1 & 0.4236 & 42.108 & $\%$ Less than 1 & Significant \\
\hline Error & 45 & 0.4527 & & & \\
\hline Total(Adjusted) & 47 & 1.0000 & & & \\
\hline
\end{tabular}

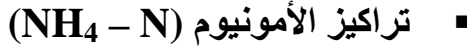

يظهر تأثير العمق كما موضح في الجدول (6) أكثر من زمن التحلل على تركيز الأمونيوم في العصـارة، حيث

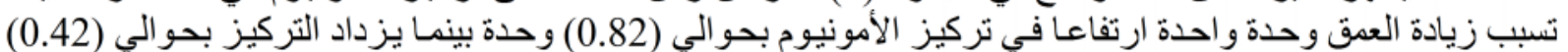
(3) النموذج الرياضي للعلاقة. كما تم إدخال البيانات في تحليل التباين

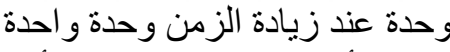
ليتضح أن المتغير زات معنوية التأثنير

(NH4-N) تأثير العمق وزمن التحلل على تركيز (6)

\begin{tabular}{|c|c|c|}
\hline \multirow{3}{*}{$\mathrm{NH}_{4}-\mathrm{N}$} & Independent variable & Standardized Coefficient $(\beta)$ \\
\cline { 2 - 3 } & Intercept & 0 \\
\cline { 2 - 3 } & depth & 0.8196 \\
\cline { 2 - 3 } & time & 0.4196 \\
\hline
\end{tabular}

$\mathbf{N H}_{4}-\mathbf{N}=-241.99+335.97 x$ depth $+2.86 x$ time $\ldots$ (3)

(NH4-N) تحليل التباين للمتغيرات ومعنوية تأثير ها على تركيز

\begin{tabular}{|c|c|c|c|c|c|}
\hline Model Term & DF & $\mathrm{R}^{2}$ & F Calculate & Prob Level & \\
\hline Model $\left(\mathrm{NH}_{4}-\mathrm{N}\right)$ & 2 & 0.8903 & 182.553 & $\%$ Less than 1 & \\
\hline Depth & 1 & 0.6692 & 274.431 & $\%$ Less than 1 & Significant \\
\hline Time & 1 & 0.1754 & 71.947 & $\%$ Less than 1 & Significant \\
\hline Error & 45 & 0.1097 & & & \\
\hline Total(Adjusted) & 47 & 1.0000 & & & \\
\hline
\end{tabular}

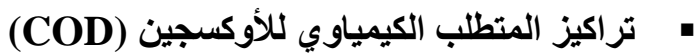

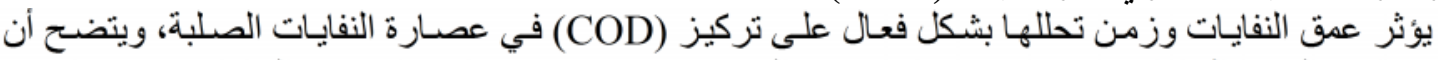

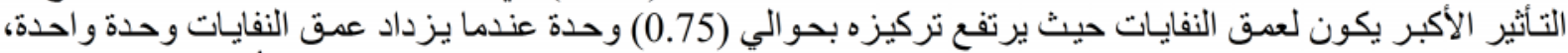

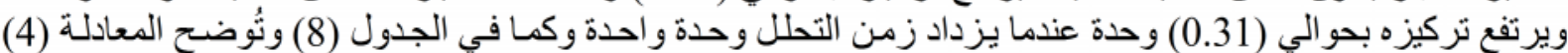

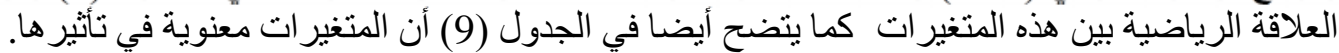


(COD) (8) (8) (الموديل الرياضي لتركيز

\begin{tabular}{|c|c|c|}
\hline \multirow{3}{*}{ COD } & Independent variable & Standardized Coefficient $(\beta)$ \\
\cline { 2 - 3 } & Intercept & 0 \\
\cline { 2 - 3 } & Depth & 0.7501 \\
\cline { 2 - 3 } & Time & 0.1312 \\
\hline
\end{tabular}

COD $=-3902.65+20502.16 x$ depth $+58.75 x$ time $\ldots$ (4)

(9OD) تحليل التباين للمتفيرات ومعنوية تأثيرها على تركيز (9)

\begin{tabular}{|c|c|c|c|c|c|}
\hline Model Term & $\mathrm{DF}$ & $\mathrm{R}^{2}$ & F Calculate & Prob Level & \\
\hline Model (COD) & 2 & 0.5846 & 31.666 & $\%$ Less than 1 & \\
\hline Depth & 1 & 0.5624 & 60.922 & $\%$ Less than 1 & Significant \\
\hline Time & 1 & 0.0172 & 1.865 & $\%$ Less than 1 & Significant \\
\hline Error & 45 & 0.4154 & & & \\
\hline Total(Adjusted) & 47 & 1.0000 & & & \\
\hline
\end{tabular}

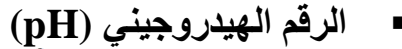

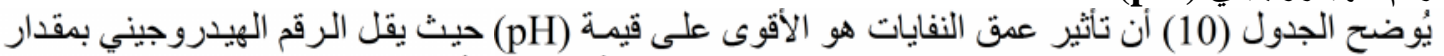

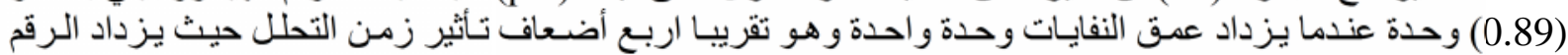

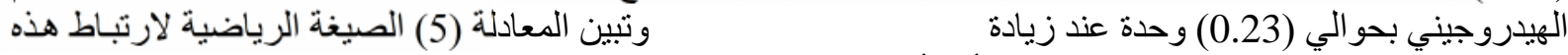
المتغير ات مع قيمة (pH). يبين الجدول (11) وحة (11) أن نأثير عمق النفايات وزمن تحلاها معنويا على الرقم الهيدروجيني.

(10) (الموديل الرياضي لقيمة (10)

\begin{tabular}{|c|c|c|}
\hline \multirow{3}{*}{$\mathrm{pH}$} & Independent variable & Standardized Coefficient $(\beta)$ \\
\cline { 2 - 3 } & Intercept & 0 \\
\cline { 2 - 3 } & Depth & $0.8866-$ \\
\cline { 2 - 3 } & Time & 0.2301 \\
\hline
\end{tabular}

pH $=7.07-1.56 x$ depth $+0.007 x$ time ... (5)

(pH) تحليل التباين للمتغيرات ومعنوية تأثير ها على قيمة

\begin{tabular}{|c|c|c|c|c|c|}
\hline Model Term & $\mathrm{DF}$ & $\mathrm{R}^{2}$ & F Calculate & Prob Level & \\
\hline Model (pH) & 2 & 0.8293 & 109.276 & \% Less than 1 & \\
\hline Depth & 1 & 0.7857 & 207.066 & $\%$ Less than 1 & Significant \\
\hline Time & 1 & 0.0529 & 13.950 & $\%$ Less than 1 & Significant \\
\hline Error & 45 & 0.1707 & & & \\
\hline Total(Adjusted) & 47 & 1.0000 & & & \\
\hline
\end{tabular}

\section{(TSS)}

يؤثر عمق النفايات بشكل أعلى من زمن التحلل كما في الجدول (12) فيرتفع تركيز المو اد العالقة بحو الي وكاي

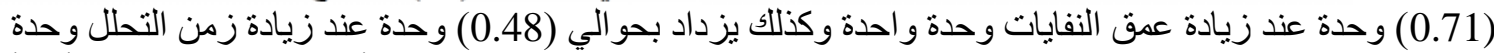

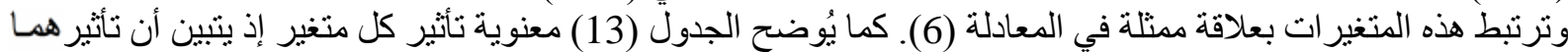
معنويا على تركيز المو اد الصلبة.

(12) الموديل الرياضي لتركيز (TSS)

\begin{tabular}{|c|c|c|}
\hline \multirow{3}{*}{ TSS } & Independent variable & Standardized Coefficient $(\beta)$ \\
\cline { 2 - 3 } & Intercept & 0.0000 \\
\cline { 2 - 3 } & depth & 0.7102 \\
\cline { 2 - 3 } & time & 0.4812 \\
\hline
\end{tabular}


$\boldsymbol{T S S}=-146.48+481.76 x$ depth $+5.31 \times$ time

(TSS) تحليل التباين للمتغيرات ومعنوية تأثيرها على تركيز

\begin{tabular}{|c|c|c|c|c|c|}
\hline Model Term & $\mathrm{DF}$ & $\mathrm{R}^{2}$ & F Calculate & Prob Level & \\
\hline Model (TSS) & 2 & 0.7701 & 75.367 & Less than $1 \%$ & \\
\hline depth & 1 & 0.5031 & 98.469 & Less than $1 \%$ & Significant \\
\hline time & 1 & 0.2310 & 45.215 & Less than $1 \%$ & Significant \\
\hline Error & 45 & 0.2299 & & & \\
\hline Total(Adjusted) & 47 & 1.0000 & & & \\
\hline
\end{tabular}

يرتبط تركيز الملوثات في عصلارة النفايات الصلبة بعلاقة طردية مباترة مع عمق هذه المبات النفايات كما يرتبط أيضا

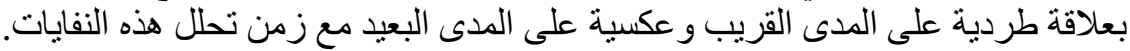

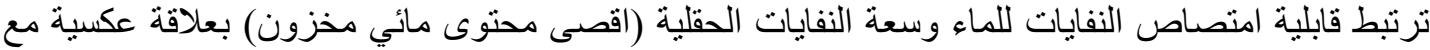

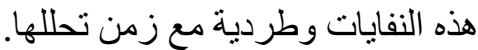

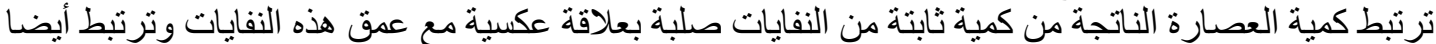

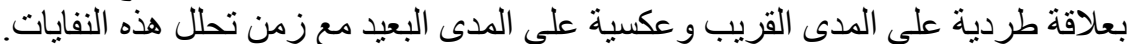

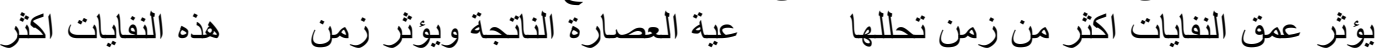
عمقها على كمية العصارة الناتجة.

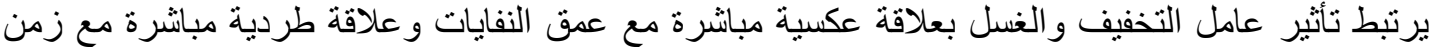
تحلل هذه النفايات، كما ترتبط سر عة التحلل أيضا بعلاقة عكسية مباشرة عـ مع عمق هذه النفايات.

[1]. Bishop, P. (2000) "Pollution prevention", fundamentals and practice, McGraw-Hill press, New York, United States Of America.

[2].Tchobanoglous, G., Theisen, H., Vigil, S.A. (1993) "Integrated solid waste management", engineering principles and management issues, $1^{\text {st }}$ Ed, McGraw-Hill press, New York, United States Of America.

[3].Mudau, A., (2012), "A Laboratory investigation of the effects of water content and waste composition on leachate and gas generation from simulated MSW", Master Thesis, Witwatersrand University.

[4].AL- Touqi, S. (2012) "Modeling Leachate BOD and COD Using Lab Scale Reactor Landfills and Multiple Linear Regression Analysis“, $\mathrm{PhD}$ Thesis, Texas University, Arlington.

[5].Imhoff, P.T., Reinhart, D.R., Englud, M., Gue' Rin, R., Gawande, N., Han, B., Jonnalagadda, S., Townsend, T.G. and Yazdani, R. (2007) "Review of state of the art methods for measuring water in landfills", Waste Manage, 27, 729-745.

[6].Kjeldsen, P.; Barlaz, M.A.; Rooker, A.P.; Baun, A.; Ledin, A.; and Christensen T.H. (2002) "Present and long-term composition of MSW landfill leachate, a review" Critical reviews in environmental science and technology, 32(4):297-336.

[7].Reinhart, D. R. and Caroline J. G. (1998) "Analysis of Florida MSW landfill leachate quality data", University of central Florida civil and environmental engineering department.

[8].Chen, P. H. (1996) “Assessment of leachates from sanitary landfills, impact of age, rainfall and treatment" Environment International, 225-22(2),

[9].Brady, N.C. and Weil, R.R. (1999), "The nature and properties of soils", 12th Edition, Prentice-Hall, Inc. New Jersey. 
[10].Kirkeby JT, Birgisdóttir H, Bhander GS, Hauschild MZ, Christensen TH. (2007) "Modeling of environmental impacts of solid waste landfilling within the life-cycle analysis program ease waste", Waste Manag. 27(7):961-70.

[11].Warith, M. (2001) "Bioreactor landfills: experimental and field results", Amsterdam, Elsevier Science Ltd.

[12].Al-Yaqout AF., Hamoda MF. (2003) "Evaluation of landfill leachate in arid climate" ,Case Study, Department of Civil Engineering, Kuwait University, Environ Int. aug,29(5):593-600.

[13].Statom, R.A., Thyne, G.D. and Mccray, J.E. (2004) "Temporal changes in leachate chemistry of a municipal solid waste landfill cell in Florida", USA, Environmental Geology volume 45, number 7 982-991, DOI: 10.1007/s00254-003-09570 .

[14].Tuffaha, B. R. (2006) "Impacts of solid waste leachate on soil and its simulation to ground water at Nablus area", (Master Thesis) Al-Najah National University, Nablus, Palestine.

[15].Esakku, S., Karthikeyan, O. P., Joseph, K., Nagendran, R., Palanivelu, K., Pathirana, K.P.M.N., Karunarathna, A.K., and Basnayake, B.F.A. (2007) "Seasonal variations in leachate characteristics from municipal solid waste dumpsites in India and Srilanka", proceedings of the international conference on sustainable solid waste management, 5 - 7 September, Chennai, India. pp.341-347341

[16].kasam , sarto, syamsiah, s., prasetyo, a., (2012), "effect of age of municipal solid waste on quantity and quality of leachate generation by column-landfill experiment", international journal of pure and applied research in engineering and technology, issn: 2319-507x, ijpret, 2012; volume 1(2): 11-21

[17].Fatima, S., Rafiq, S. K., Hai, A. (2012) "Effect of depth and age on leachate characteristics of Achan landfill, Srinagar, Jammu and Kashmir, India", Iosr journal of environmental science, toxicology and food technology (iosr-jestft) ISSN: 2319-2402, isbn: 2319-2399. volume 2, issue 2, pp 04-11

[18].Al- Rawi, S.M. (2007) "Selection, design, and management of solid waste sanitary landfill site(s) for Mosul City", Arab science and technology foundation (ASTF), project en 20 contract no. 27-06.

[19].Pheleps H., Heinke G., Jonkers J., Ouana E., and Vandecasted C. (1996) "Management of solid waste", Unesco-Col publication, Paris.

[20].Vesilind, Aarne P., Worrell, W. and Reinhart D. (2002) "Solid waste engineering", United States of America, Brooks/Cole.

[21].McGhee, T.J.; Steel, E.W. "Water Supply and Sewerage", McGraw hill International Book Company, Inc., New York. (1991)

[22].Landva, A. O. and Clark, J. I. (1990) "Geotechnics of waste fill - theory and practice", ASTM, Stp 1070. ASTM. Philadelphia, pa., pp. 86-106.

[23].Berge N.D., Reinhart D.R., Townsend T.G. (2005) "Fate of nitrogen in bioreactor landfills", Crit. Rev. Env. Sci. technol. 35, 365-399.

The work was carried out at the college of Engineering. University of Mosul 